\title{
Process management in hospitals: an empirically grounded maturity model
}

\author{
Anne Katharina Cleven · Robert Winter • \\ Felix Wortmann - Tobias Mettler
}

Received: 13 March 2013/Accepted: 26 May 2014/Published online: 5 August 2014

(C) The Author(s) 2014. This article is published with open access at Springerlink.com

\begin{abstract}
In order to improve transparency and stabilise health care costs, several countries have decided to reform their healthcare system on the basis of diagnosisrelated groups (DRG). DRGs are not only used for classifying medical treatments, but also for case-based reimbursement, hence induce active competition among hospitals, forcing them to become more efficient and effective. In consequence, hospitals are investing considerably in process orientation and management. However, to date there is neither a consensus on what capabilities hospitals need to acquire for becoming process-oriented, nor a general agreement on the sequence of development stages they have to traverse. To this end, this study proposes an empirically grounded conceptualisation of process management capabilities and presents a staged capability maturity model algorithmically derived on the basis of empirical data from 129 acute somatic hospitals in Switzerland. The five capability maturity levels start with 'encouragement of process orientation' (level 1), 'case-bycase handling' (level 2), and 'defined processes' (level 3). Ultimately, hospitals can reach the levels 'occasional corrective action' (level 4) and 'closed loop improvement' (level 5). The empirically derived model reveals why existing, generic capability maturity models for process management are not applicable in the hospitals context: their comparatively high complexity on the one hand and their strong focus on topics like an adequate IT integration and process automation on the other make them inadequate for solving the problems felt in the hospital sector, which are
\end{abstract}

Responsible editor: Thomas Hutzschenreuter (Management).

A. K. Cleven $\cdot$ R. Winter $(\bowtie) \cdot$ T. Mettler

Institute of Information Management, University of St. Gallen, Müller-Friedberg-Strasse 8, 9000 St.

Gallen, Switzerland

e-mail: robert.winter@unisg.ch

F. Wortmann

Institute of Technology Management, University of St. Gallen, Dufourstrasse 40a, 9000 St. Gallen, Switzerland 
primarily of cultural and structural nature. We deem the proposed capability maturity model capable to overcome these shortcomings.

Keywords Health care management · Process management · Maturity model $\cdot$ Design science research

JEL $\quad \mathrm{H} 75 \cdot \mathrm{I} 18 \cdot \mathrm{L} 31 \cdot \mathrm{M} 15$

\section{Introduction}

The Swiss health care system takes a leading position among the member states of the Organisation for Economic Co-Operation and Development (OECD). Its high performance is reflected in an above average life expectancy (81.9 years, birth in 2007; OECD 2010) as well as the numbers of primary care doctors and hospital beds per resident, both being among the highest of all industrialised countries (OECD 2010). However, Switzerland also faces significant health care expenditures: in 2007 the costs of health care accounted for $10.8 \%$ of the gross domestic product (OECD 2010). As a consequence, the Swiss health care system has-like that of many other OECD countries - turned into a burden for the national economy (Herzlinger 2007). In an attempt to improve transparency and stabilise health care costs, the Swiss parliament decided to substitute the existing system by a fixed-fee prospective payment based on diagnosis-related groups (DRG) from 2012 on (SwissDRG 2011). Instead of receiving a cost-based reimbursement for treatments, medications and other resources, Swiss hospitals will earn a fixed fee per case based on a predefined catalogue (SwissDRG 2011). The introduction of the DRG reimbursement scheme encourages transparency and comparability of costs as well as quality of inpatient care (Dormont et al. 2006: 31). Accompanying the pressure induced by the initiation of DRG, economic pressure and new legislation force hospitals to be highly resourceefficient, to comply with numerous security standards, to improve the quality of patient care, and to better meet the need of patients (van Oostenbrugge et al. 2009: 5).

In view of these developments hospitals can no longer act as organisations whose funding and institutional survival is "a given" and thus secure. With the introduction of DRG Swiss hospitals-like other hospitals in Europe and the US - cannot count anymore on full cost recovery. Thus, investing significantly in means to streamline operations while ensuring a high quality of patient care is becoming more important (Helfert 2009: 937). In industries such as manufacturing, finance, and logistics, the adoption of business process management (BPM) has long been the answer to increased competition and institutional pressure (Zairi 1997: 66). A substantial body of research confirms the aptitude of BPM for gaining and sustaining efficiency and competitive advantage (Kohlbacher and Reijers 2013; Skrinjar et al. 2008). Stimulated by the success experienced in other industries, organisational models such as the patient-focused hospital or clinical pathways have been developed to introduce process management into hospitals (Bragato and Jacobs 2003; Hurst 1996; Rohner 2012). However, the implementation of processoriented paradigms in the hospital context can be a considerable challenge and 
involves overcoming a number of obstacles (Hellström et al. 2010). These include a distinct functional division of labour and incentive mechanisms rewarding conformity with professional rather than institutional values (Vera and Kuntz 2007: 58). That means that "local", functional goals and norms have higher impact on organisational practice than organisation-wide, institutional goals and norms.

Process orientation-especially in healthcare-cannot be achieved in a "big bang" (McNulty and Ferlie 2004). Capabilities need to be developed stepwise in order to allow a sustained adaptation of work practices that might be fundamental (Mettler 2011b). In addition, the scope of process orientation adoption is not limited to technology (e.g. workflow support software tools), organisation (e.g. clinical pathway specifications), or people (e.g. developing collaboration across functional silos)_-process orientation needs to integrate all these capability areas (Reijers and Liman Mansar 2005). As a consequence, an organisational development process needs to be planned and controlled that might comprise several stages (a large shift can mostly not be achieved in one effort) and that integrates a diverse set of capability areas (strategy, structures, processes, organisational culture, etc.) in a coherent way. Most traditional (re-)design instruments focus on one development stage and/or one capability area. A prominent exemplar is Business Process Reengineering (Hammer and Champy 1993). For a complex, multi-faceted development process, a capability maturity model (CMM) appears to be more appropriate. First, CMMs integrate diverse notions of an evolutionary process by aggregating them into coherent stages, so-called maturity levels. Second, CMMs provide guidance on which capabilities to develop in a meaningful sequence (Becker et al. 2009: 213). Third, a multitude of otherwise incompatible ideas can be coherently covered by the unifying concept of maturity.

CMMs have become widely discussed and applied with the pioneering CMM for software development (Paulk et al. 1993). As a consequence, the term CMM is often associated with that specific field. In this article, we use the acronym CMM for any capability maturity model, regardless of its application field.

A CMM usually comes together with an instrument that supports to assess the asis state of the organisational artefact under development. By prescribing which capabilities are implying higher maturity stages, a CMM can also support to-be design of the organisational artefact. From comparing to-be and as-is maturity, a staged development plan can be derived that prescribes which capabilities need to be acquired in which sequence.

To date, however, there is neither a consensus on what capabilities hospitals need to acquire for becoming process-oriented nor a general agreement on the series of development stages they have to traverse. In fact, research has yet to develop an understanding of both the required capabilities for and maturity levels of process management in hospitals. This article therefore addresses the following research questions:

1. What process management capabilities are relevant for hospitals?

2. What stages of process management evolution have to be traversed in hospitals?

In order to strengthen the justification of the proposed CMM, we aim at conceptualising process management capabilities in an empirically grounded way. 
Additionally, the stages of process management evolution shall be algorithmically derived on the basis of empirical data instead of being "defined" using generic level characteristics like "measured" or "self-optimizing".

Our work builds upon prior statistical research affirming that process orientation significantly enhances hospital performance (Cleven et al. 2011). Overall, we see our research as a contribution towards a deeper understanding of process management maturity in hospitals. Rather than focusing on a specific facet, we build upon latest process management research suggesting that diverse capability areas covering a wide range of business, technical as well as people-related aspects need to be considered (Cleven et al. 2011). Thereby, we present a CMM that aims at providing a consistent design and management view on the multifaceted subject at hand.

The remainder of this article is structured as follows. The subsequent section sets the foundations and presents the identified research gap. Then a description of the research method, the conceptualisation of relevant capabilities and the data-driven design of the CMM is presented. This is followed by the discussion of the resulting theoretically grounded artefact. A short reflective discussion on implications, limitations and future research concludes the article.

\section{Foundations}

\subsection{Research on stages of development and maturity}

The development of organisational capabilities and the dynamics of organisational evolution have intrigued both researchers and practitioners since the advent of corporate ventures. Over time a multitude of theories that aim at explaining and predicting patterns of organisational change have been developed, including life cycle theory, teleology, dialectics, and evolution (Gardner 1965; de Ven et al. 1995: 520). Especially life cycle theory is closely related to the concept of maturity models in that both describe a typical pathway of change based on distinct stages of development (Fraser et al. 2002: 244). Maturity models also have been related to the theory of dynamic capabilities (Teece et al. 1997), seeing them as a central instrument for identifying major gaps between the enterprise and the ever-changing business environment and as important boundary object for the development and renewal firm resources (Killen et al. 2008; Klievink and Janssen 2009; Škrinjar and Trkman 2013).

The purpose of a CMM denotes the major type of recommendation it offers, that is, descriptive, prescriptive or comparative information. Descriptive CMMs are diagnostic in nature and portray evolution patterns empirically observed in a number of organisations at a certain point in time, whereas prescriptive models offer guidance for capability improvement and comprise detailed counselling. Comparative models, in turn, provide means for juxtaposing an organisation's own with other organisations' maturity levels (van Steenbergen 2011: 113).

Maturity is in the context of maturity modelling understood as a "measure to evaluate the capabilities of an organisation" (Rosemann and Bruin 2005: 1). CMMs 
allow for the assessment of maturity of a variety of different items, e.g. technologies and/or systems (e.g. Popovic et al. 2009), processes (e.g. Rosemann and Bruin 2005) or skills (e.g. Curtis et al. 2010). If many items are considered to be relevant that somehow belong together or that can be attributed to a particular worldview, they are grouped into capability areas and ultimately into "factors" (Rosemann and Bruin 2005; Rosemann and Brocke 2010). For instance, capabilities that relate to hard and soft skills of employees can be labelled as people capability area (see Sect. 3.1). It is important to notice that, although maturity models with only one capability area exist, it has become a common practice today to use a cumulative set of different capability areas (van Steenbergen 2011: 114). If the number of relevant items is not too high, it might be sufficient to differentiate two granularity layers (items/capabilities vs. capability areas) instead of three (items, capability areas and factors).

Another fundamental construct in CMM are levels. They represent archetypal states of maturity of the characteristic that is assessed. Each maturity level "represents a distinctive evolutionary plateau" (Dekleva and Drehmer 1997: 95), providing a performance description at different levels of granularity (Fraser et al. 2002: 246). In our previous example, possible levels of maturity for the "process education" characteristic allocated in the people capability area could range from an initial level "no formal approach" to a final level "continuous improvement emphasised". Also here it is important to notice, that different possibilities for defining and naming the discrete levels of maturity exist. A profound empirical study by Fraser et al. (2002) showed that most CMM used 3-6 levels for delineating an evolutionary development path.

With regard to the development of CMMs the top-down and the bottom-up approach can be distinguished (Lahrmann et al. 2011: 177). While the top-down approach specifies that levels be defined first and thereafter completed with characteristics describing the different capability areas, the bottom-up approach prescribes that capability areas and characteristics be derived first and then assigned to different maturity levels.

Moreover, three maturity principles can be discriminated: staged, continuous and area-based. While staged CMMs require all elements of one distinct level to be achieved in all capability areas, continuous models allow characteristics to be scored at different levels (Fraser et al. 2002: 244-245). In other words: in order to reach a certain level in a staged CMM, it is compulsory to comply with all requirements in all capability areas of that level. In a continuous CMM, intermediate maturity levels (which can be defined "between" two basic maturity levels) can be reached by reaching only a subset of requirements. Maturity can not only be measured in total, but also for each capability area (van Steenbergen 2011: 109).

\subsection{Process management CMMs for hospitals}

The last 2 decades saw great interest in BPM as a management concept. Its early foundations had already been laid in the 1920s with the development of scientific management, but it only became entirely practicable with the introduction of IT in 
the 1980s and 1990s (Bonham 2008; Davenport 1993; Hammer 1990). Despite the omnipresent interest in the phenomenon, researchers and practitioners have not yet agreed upon a common definition. Following Michael Hammer-one of the concept's intellectual fathers-BPM is defined as "a comprehensive system for managing and transforming organisational operations" (Hammer 2010: 3). The concept of BPM builds upon the notion of a business process as a series of crossfunctional activities that need to be performed in order to collectively achieve a predefined goal (Davenport 1993; Hammer 1990).

A number of models to assess the maturity of BPM have been developed over recent years (Rosemann and Bruin 2005: 3; Rosemann and Brocke 2010: 109). Among these a great plenty is based on the well-known Capability Maturity Model Integrated developed by the Software Engineering Institute at Carnegie Mellon University for assessing the maturity of software development processes (e.g. Fisher 2004; Rohloff 2009). Maturity in these models is defined and measured in different ways: maturity definitions include for instance effectiveness and efficiency, while maturity measurement differentiates subjective or objective measures (Rosemann and Brocke 2010: 111). Existing CMMs can further be distinguished into those that regard instances of specific process types as the object of maturity assessment and those that aim at the maturity evaluation of BPM as a holistic management approach (Pöppelbuß and Röglinger 2011: 343).

Following the search practices suggested by vom Brocke et al. (2009) and Fettke (2006), we conducted an extensive literature search so as to identify potentially available BPM CMM for the hospital sector. In order to cover the most relevant IS and health care outlets, like journals, books, conference proceedings, and practitioner magazines, the scholarly databases ScienceDirect, Proquest, EBSCOhost, and PubMed were included in the search. The search strings used were: (1) 'maturity model', (2) 'life cycle', (3) 'business process management', (4) 'health care', (5) 'hospital', and (6) 'clinical path'. Keywords were combined with following logic: (1 OR 2) AND (3 OR 4 OR 5 OR 6). The query covered all available journals, without a limitation to the field of IS. The keyword combination was required to appear in the title, the subject or keywords, and the abstract of each article. However, despite the comprehensiveness of the search, CMMs that are specifically dedicated to the assessment of process management in hospitals were not found and are not available to date to the best knowledge of the authors.

\subsection{Research gap}

It is not entirely unexpected that no domain-specific maturity model was found, as it coincides with frequent criticism pertaining to existing BPM CMM: these are found to be almost identical and hardly differing with regard to their scope, domain focus, and audience (Plattfaut et al. 2011: 328). Another frequently raised objection concerns the inconsiderate use of extant models in new application domains and the associated negligence of organisation- and industry-specific characteristics (Mettler and Rohner 2009: 3). McCormack et al. further remark that most available models mainly rely "upon anecdotal evidence and case studies describing success stories" (2009: 793) and lack an adequate theoretical basis. The same applies to reliable and 
comprehensible CMM development techniques, which to date represent an exception (Lahrmann et al. 2011: 177).

At first sight, one may think that adopting an existing BPM CMM for the hospital sector is a valid option for the paper at hand. However, existing BPM maturity models stem from manufacturing and service industries. In accordance with contingency theory (Donaldson 2006) we decided against adoption. There is no "one size fits all" BPM, i.e. hospitals are significantly different: today, hospitals are mostly characterised as loosely coupled sets of highly specialised silos with partly dubious incentive systems and an intense shielding of medical groups (Vera and Kuntz 2007: 64). Consequently, their way towards a successful process management requires a much stronger focus on both cultural and structural capability areas than it does in manufacturing and service organisations, where the focus is rather on ITsupport and process automation.

We position our work in the contexts of design science research (March and Smith 1995) and as such is concerned with the "the systematic creation of knowledge about, and with, design" (Baskerville 2008: 441). Special emphasis is placed on the construction of sufficiently new or decisively better artefacts, constituting the "possibly sole, or chief, output of the research" (Gregor and Jones 2007: 318).

\section{Model development and research approach}

\subsection{Overview}

The overall research approach follows three major steps: in view of the lack of wellfounded and multi-faceted conceptualisations of 'maturity', we first derive the relevant capability areas for process management in the context of hospitals in Sect. 3.3. Both Lahrmann et al. (2011: 177) and Mettler (2011a: 78) name a poor if not missing theoretical basis as one of the major shortcomings of currently available CMMs. By drawing upon broadly accepted organisational and information systems (IS) theories for the conceptualisation of process management maturity, we aim at providing a rigorous foundation for the development of the model proposed in this study.

Second, in Sect. 3.4 we describe how we identify key assessment items and check their relevancy for the hospital setting.

Third, we used these verified items as a basis for deriving an assessment questionnaire which in turn is used for a data-driven determination of the CMM (Sect. 3.5) by defining maturity levels, assigning capabilities (items) to maturity levels, and thereby implying improvement paths.

However, as a precondition for a systematic model construction, the model's scope and boundaries have to be defined first in the following Sect. 3.2. 


\subsection{Scope of the proposed model}

A very recent proposal by Pöppelbuß and Röglinger offers a set of design principles to assist researchers in "maturity model design and substantiation" (2011: 345). Besides its function as a checklist or documentation template when designing MMs, it serves as an instrument to evaluate and compare alternative models. The framework is herein employed to define the scope and set the boundaries of the model to be proposed (Table 1), whereas especially the employed maturity concept as well as the algorithm for model development are subsequently described in greater detail.

\subsection{Conceptualisation of process management capability areas}

Having emerged only about two decades ago, BPM has just recently found entrance to theory-driven research. Rigorous theory for describing and explaining the phenomenon has thus not yet been developed (Houy et al. 2010; Smart et al. 2009; Trkman 2010). While some existing theories have been proposed to theoretically ground BPM (e.g. the task-technology-fit theory; Trkman 2010: 126), no broad consensus has been achieved so far. Ko et al. characterise process management as a cross-disciplinary approach that adopts a "variety of paradigms and methodologies" (2009: 745). Adequately depicting process management maturity thus calls for a conceptualisation that is capable of capturing this variety.

Soanes and Stevenson define maturity as a "state of being complete, perfect, or ready" or the "fullness of development" (2008: 906). In order to cover capability areas that establish a fullness of development in process management, we draw upon the constructs proposed in the socio-technical theory on the one hand and the organisational culture theory on the other. Socio-technical theory proposes that effectively and efficiently designing organisational systems requires taking into account both the social and the technical subsystem (Bostrom and Heinen 1977: 14). The same holds true for BPM: while the approach intends to increase the performance of an organisation through breaking functional walls and streamlining work, it only became truly practicable with the introduction of information technology (IT) (Bonham 2008: 125). According to socio-technical theory, the technical system comprises the two components technology and tasks (Bostrom and Heinen 1977: 25). Stemming from the Greek téchne, the technical system is concerned with "processes, tasks, and technology needed to transform inputs to outputs" (Bostrom and Heinen 1977: 17). For the development of our MM, we have translated these concepts into the process management capability areas $I T$ and practices.

In the previous section, we outlined why hospitals need to pay close attention to both cultural and structural dimensions on their way towards successful process management. Therefore, we build upon organisational culture theory as it facilitates a deeper understanding of organisations going beyond structural considerations. Organisational culture theory differentiates the three constructs assumptions, espoused values and artefacts (Hatch 1993: 956). Assumptions represent the most intangible construct that comprises beliefs and ways of interpersonal communication and behaviour. For the CMM presented in this study, we termed this construct culture. Espoused values are goals, strategies and standards, which are condensed 


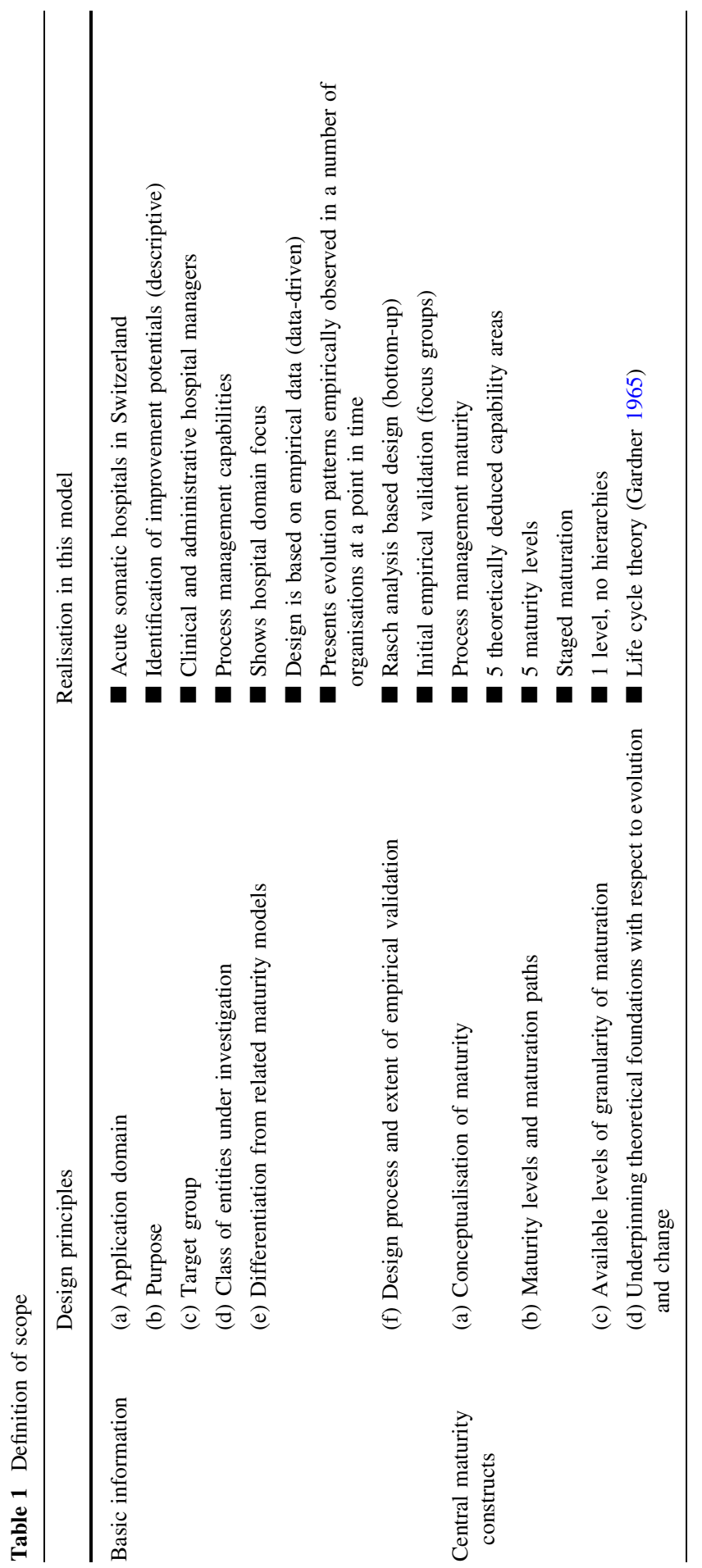




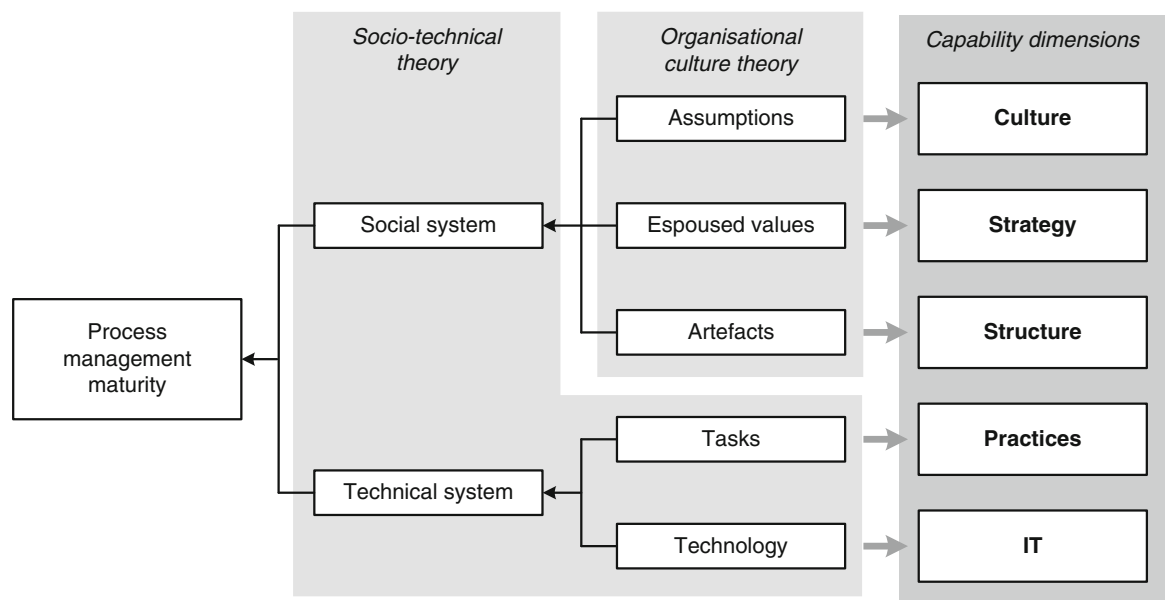

Fig. 1 Process management capability areas

under the term strategy as the fourth capability area of our CMM. Structure represents the fifth and last capability area of process management maturity derived from the original construct artefacts, which covers tangible and visible organisational structures like, e.g. departments. Both theories postulate that their respective constructs are interdependent and should be mutually aligned in order to maximise organisational benefits. The conceptual basis for our CMM is thus formed by five capability areas: culture, strategy, structure, practices, and IT (Fig. 1).

Table 2 provides a description for each capability area, while single items are introduced in the course of the further analysis.

\subsection{Identification and validation of key assessment items}

As a subsequent step, a comprehensive literature analysis was conducted in order to identify relevant work practices, principles and activities for each of the five previously identified capability areas. In order to argue about the comprehensibility, completeness, and relevancy of the identified items, we conducted several focus group discussions. Focus groups is a qualitative research method for the exploration of people's frameworks of understanding (Carter and Henderson 2005) and as such has proven to be particularly useful for investigating new ideas and to check the applicability of a research object by practitioners (Chiarini Tremblay et al. 2010). Between 2009 and 2010, a total of 12 sessions with distinct experts and decisionmakers from inside and outside the hospital were conducted (see Table 3). Judgment sampling was used in order to identify and select relevant stakeholders (Marshall 1996). Each session lasted at least 3 h. In the first sessions, we asked the experts about the inclusion or exclusion of items, the allocation of items to the identified capability areas, and the structuring of the questionnaire. In the last sessions, we asked the experts to discuss the applicability of the conceptual framework as well as the relevancy for their work. 


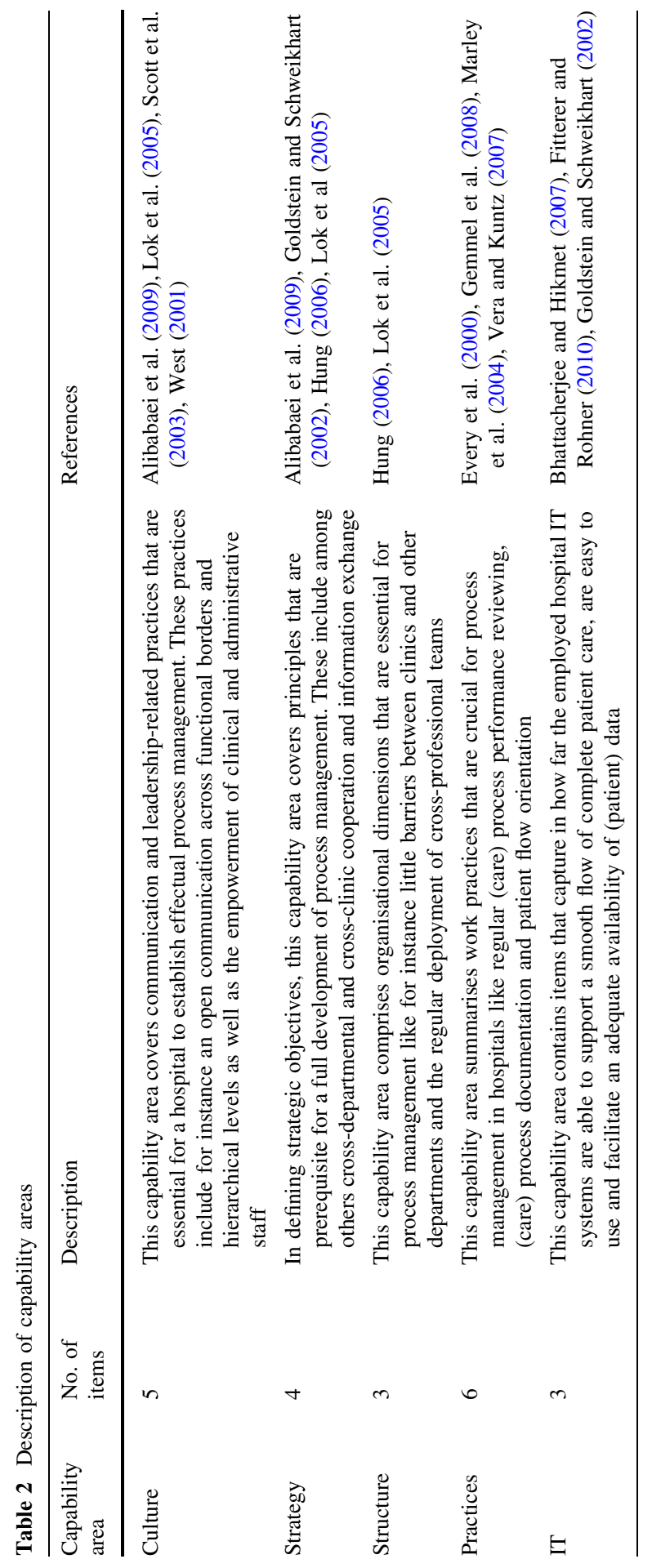


Table 3 Members of the focus group

\begin{tabular}{|c|c|c|c|}
\hline $\begin{array}{l}\text { Number } \\
\text { of experts }\end{array}$ & Institution & Role & Experience \\
\hline 1 & $\begin{array}{l}\text { Basic-care hospital (relatively } \\
\text { large/specialised) }\end{array}$ & $\begin{array}{l}\text { Senior } \\
\text { partner }\end{array}$ & $\begin{array}{l}20 \text { years experience in hospital and medical } \\
\text { management }\end{array}$ \\
\hline 1 & $\begin{array}{l}\text { Centralised care (university } \\
\text { hospital) }\end{array}$ & $\begin{array}{l}\text { Senior } \\
\text { partner }\end{array}$ & 25 years experience in hospital management \\
\hline 1 & $\begin{array}{l}\text { Basic-care hospital (middle size/ } \\
\text { specialised) }\end{array}$ & $\begin{array}{l}\text { Junior } \\
\text { partner }\end{array}$ & 10 years experience in hospital management \\
\hline 1 & $\begin{array}{l}\text { Consulting company specialised } \\
\text { in healthcare management }\end{array}$ & $\begin{array}{l}\text { Senior } \\
\text { partner }\end{array}$ & 20 years experience in hospital management \\
\hline 2 & Cantonal Health Department & $\begin{array}{l}\text { Senior } \\
\text { partner }\end{array}$ & $\begin{array}{l}25 \text { years in experience in hospital } \\
\text { organisation, payment schemes and } \\
\text { regulation }\end{array}$ \\
\hline
\end{tabular}

\subsection{Data-driven determination of improvement path}

Based on the identified capability areas and associated items, the maturity model is now constructed by defining maturity levels, assigning capabilities (items) to maturity levels, and thereby implying improvement paths. In contrast to existing CMMs which mostly define maturity levels generically ("measured", "selfoptimizing") and assign items intuitively to such levels, we aim at using a quantitative technique to develop an empirically grounded CMM. Following Dekleva and Drehmer (1997: 96 ff.) which are supported by various real-world examples (e.g. Lahrmann et al. 2011; Mettler 2011b), the Rasch algorithm has proven to be a useful analytical method to determine an evolutionary improvement path. The fundamental idea behind Rasch is that each item included in the questionnaire can be associated with a specific "difficulty" (to achieve the respective capability) and thus the items can be ordered according to this difficulty-and their "difficulty sequence" represents an empirically justified evolution. The algorithm is based on the proposition that highly skilled entities have a high probability of having successfully implemented easy items (Bond and Fox 2007: 37). By counting the answers that indicate the presence of capabilities, the algorithm calculates two scores: one for the difficulty of items and one for the ability of the surveyed entities. Both scores are measured on the same interval scale, which allows for estimating the likeliness, with which a certain entity masters a certain item. For evaluating the quality of the model, two statistics termed 'Infit' and 'Outfit' are used. Both assess whether data that have been analysed (items and survey participants) fit the expectations specified in the model. Applied in the context of CMM development, the Rasch analysis allows for the inductive allocation of items onto maturity levels based on the measurement of item difficulty as well as the assessment of surveyed entities based on their capability level (Dekleva and Drehmer 1997: 97).

Tailoring the Rasch analysis for CMM development requires some slight modifications of the basic model (Lahrmann et al. 2011: 182). Because rating scales have a stronger expressive power, five-tired Likert scales are employed instead of 
the originally proposed dichotomous scales. Conducting the Rasch analysis yields a single ordinal scale that represents the logit measure of each item and entity, but no distinct maturity levels. In order to avoid subjectivity in defining maturity levels, the modified Rasch analysis thus employs cluster analysis based on the item logits. Since most CMMs use five maturity levels (van Steenbergen 2011: 90), the anticipated number of clusters is set to five. The BIGSTEPS software, Version 2.82 (Linacre et al. 1998) was used to calculate the Rasch item calibration.

In order to obtain the necessary data for identifying different stages of capability of process management in hospitals, we distributed our previously developed questionnaire to 319 clinical and administrative hospital managers, covering all acute somatic hospitals in Switzerland that are directly affected by the introduction of DRG. The target population consisted of clinical and administrative hospital managers, who have a profound cognizance of their institutions' process landscapes. All of them are responsible for daily business as well as for organisational change initiatives in their respective institutions. Accordingly, respondents were capable of providing valid information regarding the object of study. The questionnaire was sent at the beginning of October 2010. By the end of January 2011 a total of 149 questionnaires had been returned, yielding a response rate of $46.7 \%$. 129 questionnaires were complete and have been regarded in the subsequent analyses.

Organisational demographics revealed that the distribution of public (73\%), nonfor-profit $(6 \%)$ and private $(21 \%)$ hospitals being part of the analysis adequately represent the given parent population of Swiss hospitals. With regard to size, $8 \%$ of the surveyed hospitals fall into the category 1-50 beds, $28 \%$ into the category 51-200 beds, $20 \%$ into the category $201-400$ beds, $16 \%$ into the category 401-600 beds, and $29 \%$ have more than 600 beds. Concerning the characteristics of care, $33 \%$ of the surveyed hospitals deliver primary health care (i.e. broad range of ambulant and inpatient treatments), $10 \%$ secondary care (i.e. partially specialised, interdisciplinary and mainly inpatient treatments), $34 \%$ tertiary care (i.e. special clinics, incl. non-somatic care), and $23 \%$ non-acute care (i.e. rehabilitation, chronic care). Personal demographics revealed that $50 \%$ of the respondents classify their job as administrative/managerial, $23 \%$ as clinical/therapeutic, $12 \%$ as consulting hospital management, and $15 \%$ as other.

Table 4 shows the results of applying the Rasch analysis ordered by the difficulty of items. The table includes levels, capability areas, item descriptions, and references as well as logit, Infit and Outfit values. Infit and Outfit statistics, used to assess if the data conforms to the model's assumptions, were tested. Values greater than 2 for either of the two statistics should not occur in more than $5 \%$ of the items. Our data set meets this quality criterion.

\section{Results}

Figure 2 illustrates the graphical representation of the staged CMM that describes the evolution of process management as it takes place in 129 participating Swiss hospitals. 


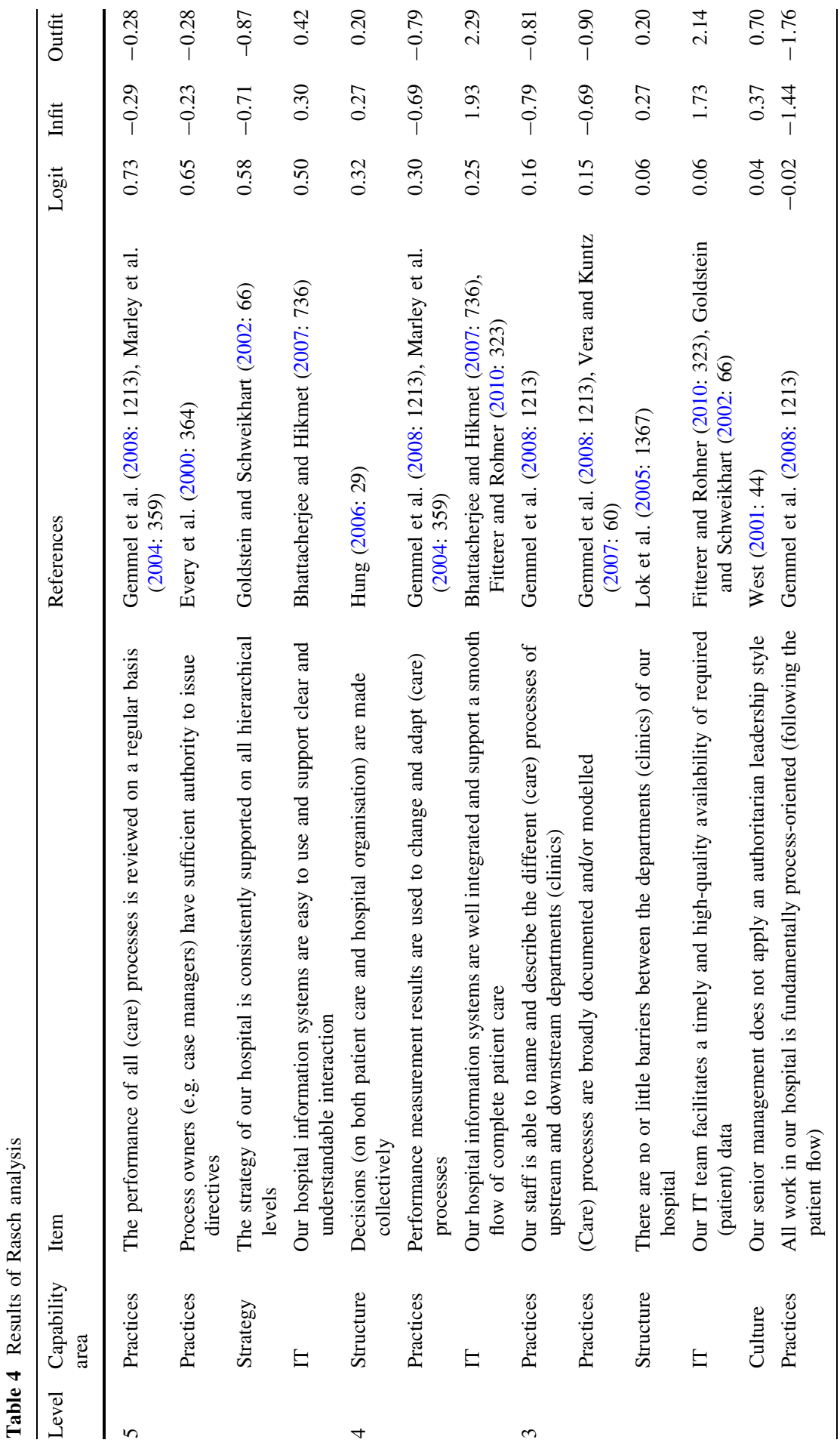




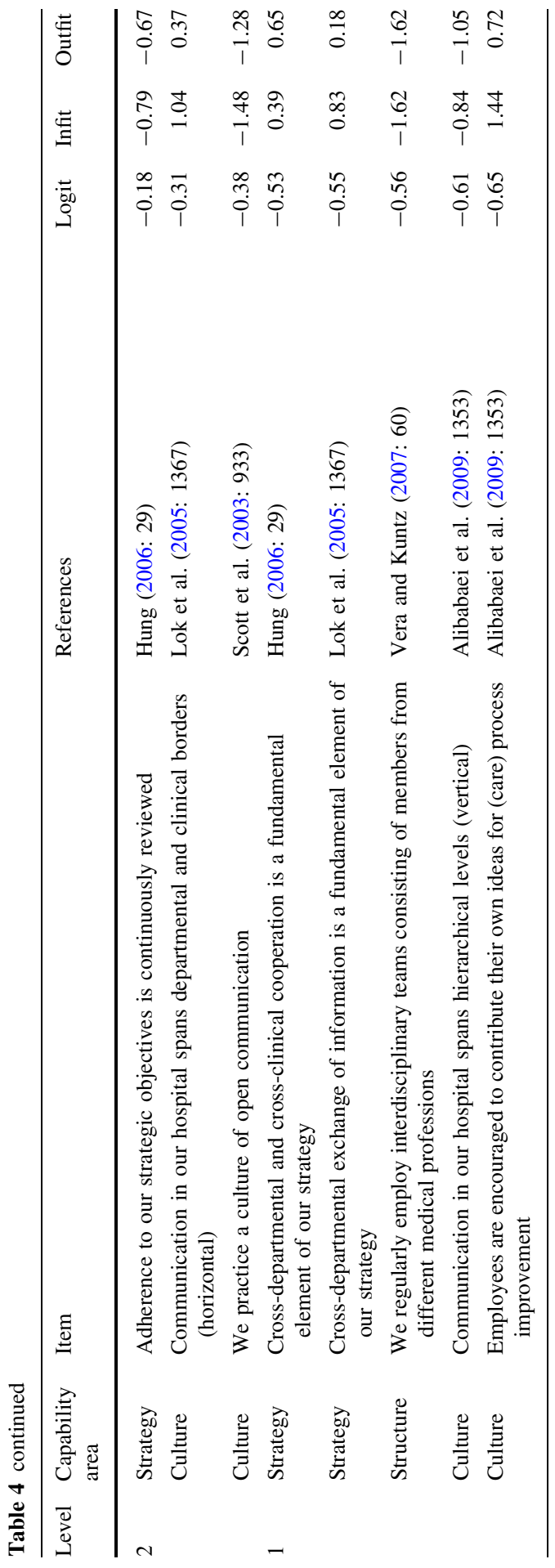


Finding names for maturity stages is an interpretive task. For CMMs that are defined "top-down" instead of empirical grounding, generic stage names like "measured" or "self-optimizing" are given by the overall approach. In our case, an analysis of the comprised capabilities yielded stage name candidates whose appropriateness was discussed within the researcher team first and subsequently with the involved experts.

Some cells of the CMM are empty owing to the fact that on "each maturity level certain components of [the object of maturity] become evident and others barely registered" (McCormack et al. 2009: 795). This is common practice in maturity model construction, as not every evolutionary step needs to cover each and every capability area (Ahern et al. 2003). In the case of the herein proposed model it becomes evident that a full implementation of process management in Swiss hospitals begins with 'setting the scene' by laying a focus on the rather soft capability areas culture and strategy in stages 1 and 2. From there on, the focus shifts to the more tangible implementation-related capability areas structure, work practices and IT. The stages of the CMM are cumulative, that is they are traversed subsequently while additively increasing the level of maturity of each capability area.

The full maturation path is outlined in the following:

\section{- Stage 1: encouragement of process orientation}

The first stage is characterised by an initial strategic commitment to process management, which is reflected in the fact that cross-clinic cooperation and information exchange represent fundamental elements of the strategy and are thus actively promoted by hospital management. While staff is encouraged to contribute ideas for improving work practices, it is not yet clear whether these ideas are actually put into practice. A regular employment of cross-professional teams also points to the appreciation of a process-oriented mode of operations.

\section{- Stage 2: case-by-case handling}

Stage two features a further movement towards process management. Open communication is actively practiced, not only between regular doctors and chief physicians of the same clinic, but also between different clinics. However, with respect to process management this stage still has an ad hoc character: while adherence to the strategic goal of implementing process management is continuously reviewed, crossdepartmental issues are in this stage only addressed in a case-by-case manner.

\section{- Stage 3: defined processes}

On stage three, process orientation spreads throughout the hospital: procedures are now modelled and documented, work steps are adjusted to follow the patient flow, and doctors and other employees are aware of the processes of up- and downstream departments and clinics. Visible and invisible barriers between departments diminish noticeably and senior management as well as chief physicians abandon their authoritarian leadership style. Clinical and administrative processes are on this stage supported by IT systems that facilitate a timely and high-quality provision of required (patient) data. 


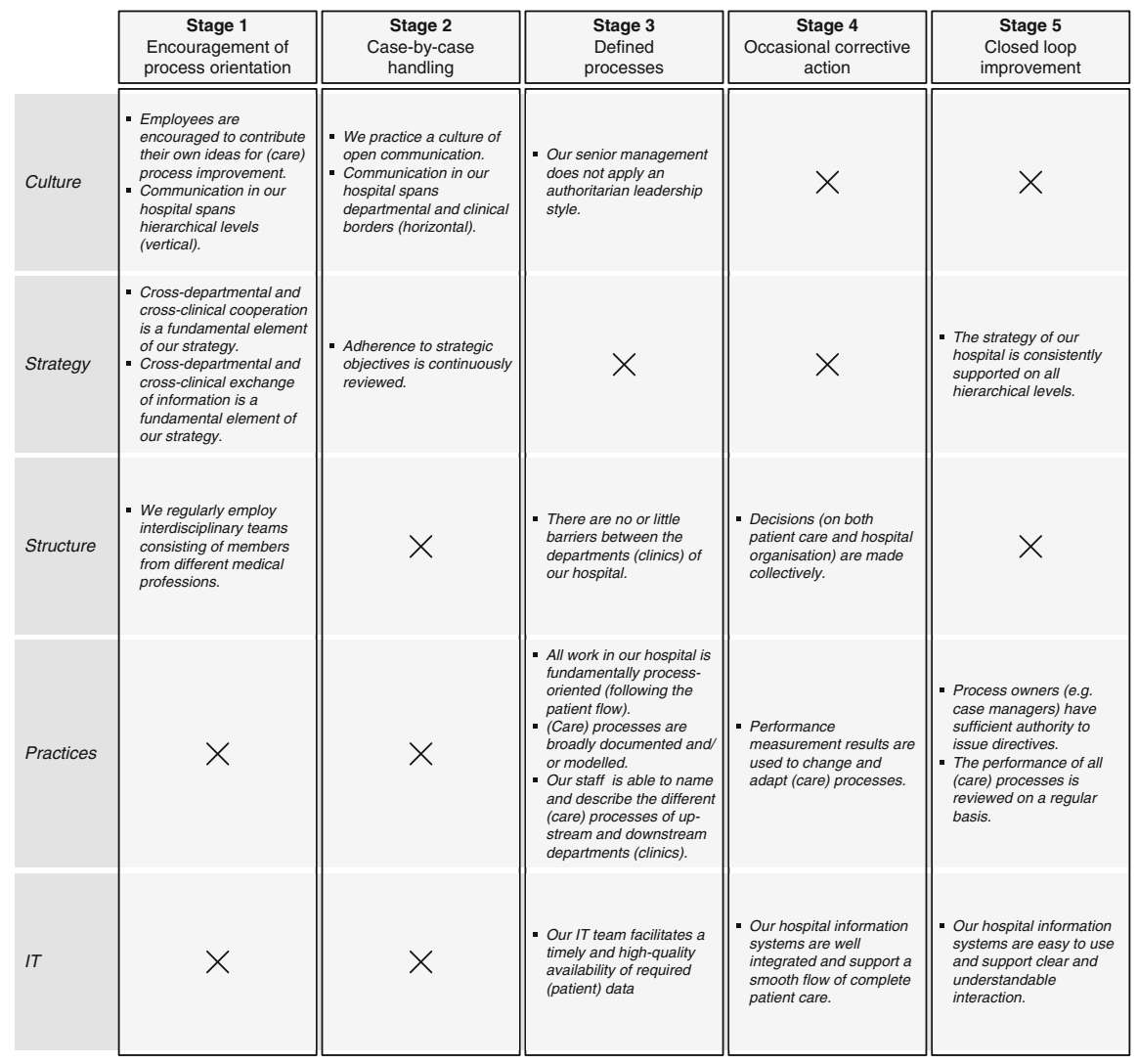

Fig. 2 A CMM for hospital process management

\section{- Stage 4: occasional corrective action}

Stage four represents a further manifestation of process management. Hospital IS are in place that are well integrated and facilitate a smooth flow of complete patient care. The performance of processes is measured on an occasional basis and-if necessary-procedures are adapted or changed. Decisions on alterations, both regarding patient care or hospital organisation, are made collectively.

\section{- Stage 5: closed loop improvement}

Stage five of process orientation in hospitals is characterised by IS that are easy to use for all staff and enable a clear and highly understandable interaction. Staff on all hierarchical levels is actively supporting the strategic decision to transform the hospital into a process-oriented organisation. At this stage, process ownership is not just a role but an established organisational entity with significant authority and process reviews are conducted on a regular basis in order to realise continuous improvement. 


\section{Critical discussion}

For a first critical discussion, the paper reflects the results on the basis of the key design science criteria 'rigor' and 'relevance' (Hevner et al. 2004: 87-88). Rigor requests that the design process and the design results are traceable, transparent, reliable, and valid (Frank 2007). Relevance is closely linked to the term utility and attends to the artefact's ability to solve the outlined problem (March and Smith 1995).

As missing methodical rigor of existing CMMs has been one motivation of this paper, the selected research method (Rasch and cluster analysis) was chosen in order to reduce potential subjectivity in CMM construction. The validity of the items has been ensured by using the existing knowledge base as well as developing and pre-testing the designed questionnaire with a focus group.

Having built the model on the basis of theory and quantitative techniques we argue that a reflection based on qualitative interviews particularly makes sense. While we consequently built upon theory and quantitative techniques, on there is a significant risk of compromising relevance. Therefore, qualitative interviews give us the chance to challenge our model on the basis of "real world", i.e. practitioner feedback. As the results of our first round of empirical evaluation revealed, it turns out to be of particular relevance and utility for the intended audiences. In order to assess the usefulness of our model, we conducted interviews with two clinical and two administrative hospital managers who had not been part of the initial survey. The interviews commenced with a brief introduction on the background and purpose of the model. Thereafter, interviewees were asked to evaluate the model with respect to the following four considerations:

- Completeness: is the model complete as relates to content?

- Utility: does it allow for determining the own position, that is for conducting a valid self-assessment?

- Utility: does the model also allow deriving means of improvement?

- Advancement: what would enhance the value of this model?

Answers are in the following presented in a summarised form. All four respondents valued the overview functionality of the model positively. Having just recently been confronted with process management as a possible answer to the increased cost containments, they appreciated the possibility to quickly obtain a grasp of what capability areas are crucial and how hospitals evolve in implementing process management. While the model was considered complete as it relates to content on the given level of granularity all interviewees remarked that a second, more detailed level would provide additional benefits. Regarding the selfassessment, all interviewees were quickly able to locate their respective hospital based on the model. Interestingly, assessments of the respective clinical and administrative managers did not differentiate much. Due to the fact that the model represents an empirically validated 'journey' towards process management, interviewees remarked that they felt able to identify necessary improvement steps based on the difference between their current position and the final stage of maturity. Thus, they attributed the model a normative character. With respect to 
utility, especially the two clinical managers remarked that-apart from the 'gap analysis' based on the maturity stages - they would appreciate additional guidance. Moreover, extending the model towards a tool for hospital-spanning assessments would be highly valuable, as one of the interviewees remarked, since the introduction of DRG also leads to a higher degree of specialisation, which again requires cooperation between hospitals. One evaluation criterion for selecting a cooperation partner may then be his level of competence with regard to process management.

\section{Conclusion}

In the light of serious cost containments and an increased competition with the introduction of the DRG-based payment system, Swiss hospitals are more than ever in need of ways to operate effectively. Process management has repeatedly been named as an effectual approach for improving quality while reducing costs and resources. In this study, we presented a theoretically grounded staged CMM for process management in hospitals based on empirical data. While the model portrays evolution patterns empirically observed in a number of hospitals at a certain point in time and is thus basically descriptive in nature, a first empirical evaluation, however, revealed that it also offers normative advice. The four evaluation partners positively valued its comprehensiveness as relates to content and the possibility to determine the own institution's position. Additional benefits may be attainable by including 'best practice' guidance and conducting organisation-spanning assessments so as to enable a well-founded benchmarking.

From a theoretical perspective, the following implications are worth mentioning. There are not many examples yet where behavioural research methods are directly employed in the context of design research, in particular for CMM construction (e.g. Marx et al. 2012). Usually, the Rasch analysis is employed to measure variables such as abilities, attitudes, and personal characteristics for psychological and educational assessments. Adapted for CMM development, the Rasch analysis allows for the inductive allocation of capabilities onto maturity levels and thereby supports the rigorous design of CMM. Moreover, through the use of cluster analysis the arbitrariness in assigning capabilities to different maturity levels that is inherent in other development methods and has been criticised by several researchers is avoided (Lahrmann et al. 2011: 177).

From a practical perspective, the following implications are worth mentioning. The model proposed in this article was specifically developed for the hospital sector. While numerous CMMs for BPM are already available, these models are highly complex. Besides, many existing models often neglect cultural and structural dimensions in favour of IT and integration support (Van Looy 2010: 693). These traits limit the utility of existing BPM CMM for the hospital sector, in which organisational considerations are the most significant: efficient structures and values as well as effectual communication capabilities are largely obsolete in hospitals characterising them as complete process management freshmen. The model introduced in this article apprehends this fact and offers a model with an adequate level of complexity that addresses the specific problems that hospitals are currently facing. 
Another question that calls for an answer is: how have hospitals that have been confronted with the DRG scheme for well over 10 years now, like, e.g. those in Germany managed the process challenge? Two recently conducted studies shed some light on this issue. Both Gemmel et al. (2008) and Vera and Kuntz (2007) investigated the maturity of process orientation and management in German hospitals. Both studies emphasise the various benefits of process orientation and management for hospitals and at the same time consistently report on a very low level of implementation. However, Vera and Kuntz (2007: 64) strengthen the necessity of a hospital-wide commitment to process orientation and the establishment of a 'process culture' as a prerequisite for a successful realisation. It is thus assumed that the model proposed in this article may also be of value for hospital management in other countries that are affected by the introduction of DRG.

Like every research, this one comes with limitations, too. By focusing our work on the multifaceted nature of process management in hospitals we had to compromise on the level of detail. Therefore, further research should focus on specific capability areas. Thereby, our model can serve as the "glue" and/or starting point for various upcoming research activities. Another limitation pertains to the evaluation of the model. While an initial evaluation has been accomplished, a broader assessment with a larger number of hospitals is indispensable. The development of the proposed model is based on a theory-led conceptualisation of maturity and a quantitative approach for determining the different stages of maturity. We consider this approach as a very promising and valuable one, but want to acknowledge that enriching it with qualitative methods may lead to an even stronger expressiveness and depth of the model. Although contextual factors (like size of the organisation, hospital type, regulatory context, strategic positioning) might influence maturity specification and maturity level definitions significantly (Raber et al. 2013), we did not consider CMM mutability so far.

Further research may apprehend and address the just-named limitations. Also, we encourage the investigation into techniques which determine the "optimal" number of maturity stages. In the paper at hand we set the number of levels ex ante to 5-in accordance to common practice. While this number is reasonable (3 or less stages hardly allow any differentiation; 7 or more stages induce certain complexity), it is also arbitrary. While the deployed clustering procedure would allow to derive consistently any other number of levels from the data set, other than only quantitative considerations should be used to determine an optimal number of levels. Another research direction should be focusing on situational CMM design and overcome the "one size fits all" assumption of our CMM. The Rasch analysis method enables the development of dedicated CMMs for specific sub-samples. Not least, investigating the actual impact of process orientation and process management on different dimensions of hospital performance and an institution's competitiveness represents an interesting avenue for further research.

Open Access This article is distributed under the terms of the Creative Commons Attribution License which permits any use, distribution, and reproduction in any medium, provided the original author(s) and the source are credited. 


\section{Appendices}

A. Questionnaire

\begin{tabular}{|c|c|c|c|c|c|c|}
\hline \multirow{2}{*}{\multicolumn{2}{|c|}{$\begin{array}{l}\text { Dimension } \\
\text { Culture }\end{array}$}} & \multicolumn{5}{|c|}{ Rating } \\
\hline & & \multicolumn{3}{|c|}{ disagree } & \multirow{2}{*}{\multicolumn{2}{|c|}{$\begin{array}{rr} & \text { agree } \\
-1 \quad+2\end{array}$}} \\
\hline & & -2 & -1 & 0 & & \\
\hline C1 & $\begin{array}{l}\text { Our senior management does not apply an authoritarian leadership } \\
\text { style. }\end{array}$ & $\square$ & $\square$ & $\square$ & $\square$ & $\square$ \\
\hline$C 2$ & $\begin{array}{l}\text { Communication in our hospital spans departmental and clinical borders } \\
\text { (horizontal). }\end{array}$ & $\square$ & $\square$ & $\square$ & $\square$ & $\square$ \\
\hline$C 3$ & Communication in our hospital spans hierarchical levels (vertical). & $\square$ & $\square$ & $\square$ & $\square$ & $\square$ \\
\hline C4 & We practice a culture of open communication. & $\square$ & $\square$ & $\square$ & $\square$ & $\square$ \\
\hline C5 & $\begin{array}{l}\text { Employees are encouraged to contribute their own ideas for (care) } \\
\text { process improvement. }\end{array}$ & $\square$ & $\square$ & $\square$ & $\square$ & $\square$ \\
\hline \multicolumn{2}{|c|}{ Strategy } & \multicolumn{2}{|c|}{ disagree } & & & agree \\
\hline & & -2 & -1 & 0 & +1 & +2 \\
\hline S1 & $\begin{array}{l}\text { The strategy of our hospital is consistently supported on all hierarchical } \\
\text { levels. }\end{array}$ & $\square$ & $\square$ & $\square$ & $\square$ & $\square$ \\
\hline$S 2$ & $\begin{array}{l}\text { Cross-departmental exchange of information is a fundamental element } \\
\text { of our strategy. }\end{array}$ & $\square$ & $\square$ & $\square$ & $\square$ & $\square$ \\
\hline$S 3$ & $\begin{array}{l}\text { Cross-departmental and cross-clinical cooperation is a fundamental } \\
\text { element of our strategy }\end{array}$ & $\square$ & $\square$ & $\square$ & $\square$ & $\square$ \\
\hline$S 4$ & Adherence to our strategic objectives is continuously reviewed. & $\square$ & $\square$ & $\square$ & $\square$ & $\square$ \\
\hline
\end{tabular}

\begin{tabular}{|c|c|c|c|c|c|c|}
\hline \multicolumn{2}{|c|}{ Structure } & \multicolumn{3}{|c|}{ disagree } & \multicolumn{2}{|r|}{ agree } \\
\hline & & -2 & -1 & 0 & +1 & +2 \\
\hline Strl & $\begin{array}{l}\text { There are no or little barriers between the departments (clinics) of our } \\
\text { hospital. }\end{array}$ & $\square$ & $\square$ & $\square$ & $\square$ & $\square$ \\
\hline Str 2 & $\begin{array}{l}\text { We regularly employ interdisciplinary teams consisting of members } \\
\text { from different medical professions. }\end{array}$ & $\square$ & $\square$ & $\square$ & $\square$ & $\square$ \\
\hline Str3 & $\begin{array}{l}\text { Decisions (on both patient care and hospital organisation) are made } \\
\text { collectively. }\end{array}$ & $\square$ & $\square$ & $\square$ & $\square$ & $\square$ \\
\hline \multicolumn{2}{|c|}{ Practices } & \multicolumn{2}{|c|}{ disagree } & & & agree \\
\hline & & -2 & -1 & 0 & +1 & +2 \\
\hline$P 1$ & (Care) processes are broadly documented and/or modelled. & $\square$ & $\square$ & $\square$ & $\square$ & $\square$ \\
\hline$P 2$ & $\begin{array}{l}\text { Process owners (e.g. case managers) have sufficient authority to issue } \\
\text { directives. }\end{array}$ & $\square$ & $\square$ & $\square$ & $\square$ & $\square$ \\
\hline$P 3$ & $\begin{array}{l}\text { Our staff is able to name and de-scribe the different (care) processes of } \\
\text { upstream and downstream departments (clinics). }\end{array}$ & $\square$ & $\square$ & $\square$ & $\square$ & $\square$ \\
\hline P4 & $\begin{array}{l}\text { All work in our hospital is fundamentally process-oriented (following } \\
\text { the patient flow). }\end{array}$ & $\square$ & $\square$ & $\square$ & $\square$ & $\square$ \\
\hline P5 & The performance of all (care) processes is reviewed on a regular basis. & $\square$ & $\square$ & $\square$ & $\square$ & $\square$ \\
\hline P6 & $\begin{array}{l}\text { Performance measurement results are used to change and adapt (care) } \\
\text { processes. }\end{array}$ & $\square$ & $\square$ & $\square$ & $\square$ & $\square$ \\
\hline \multirow{2}{*}{\multicolumn{2}{|c|}{ Information Technology }} & \multicolumn{2}{|c|}{ disagree } & & \multicolumn{2}{|c|}{ agree } \\
\hline & & -2 & -1 & 0 & +1 & +2 \\
\hline ITI & $\begin{array}{l}\text { Our hospital information systems are easy to use and support clear and } \\
\text { understandable interaction. }\end{array}$ & $\square$ & $\square$ & $\square$ & $\square$ & $\square$ \\
\hline IT2 & $\begin{array}{l}\text { Our IT team facilitates a timely and high-quality availability of } \\
\text { required (patient) data. }\end{array}$ & $\square$ & $\square$ & $\square$ & $\square$ & 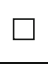 \\
\hline IT3 & $\begin{array}{l}\text { Our hospital information systems are well integrated and support a } \\
\text { smooth flow of complete patient care. }\end{array}$ & $\square$ & $\square$ & $\square$ & $\square$ & $\square$ \\
\hline
\end{tabular}


Reliability and separation of measures Rasch analysis

Following Linacre et al. (1998) person separation $(<2$, person reliability $<0.8)$ with a relevant hospital sample implies that the instrument may not be not sensitive enough to distinguish between high and low performers. Low item separation $(<3=$ high, medium, low item difficulties, item reliability $<0.9)$ implies that the hospital sample is not large enough to confirm the construct validity of the instrument. Both person and item separation fulfil the mentioned criteria.

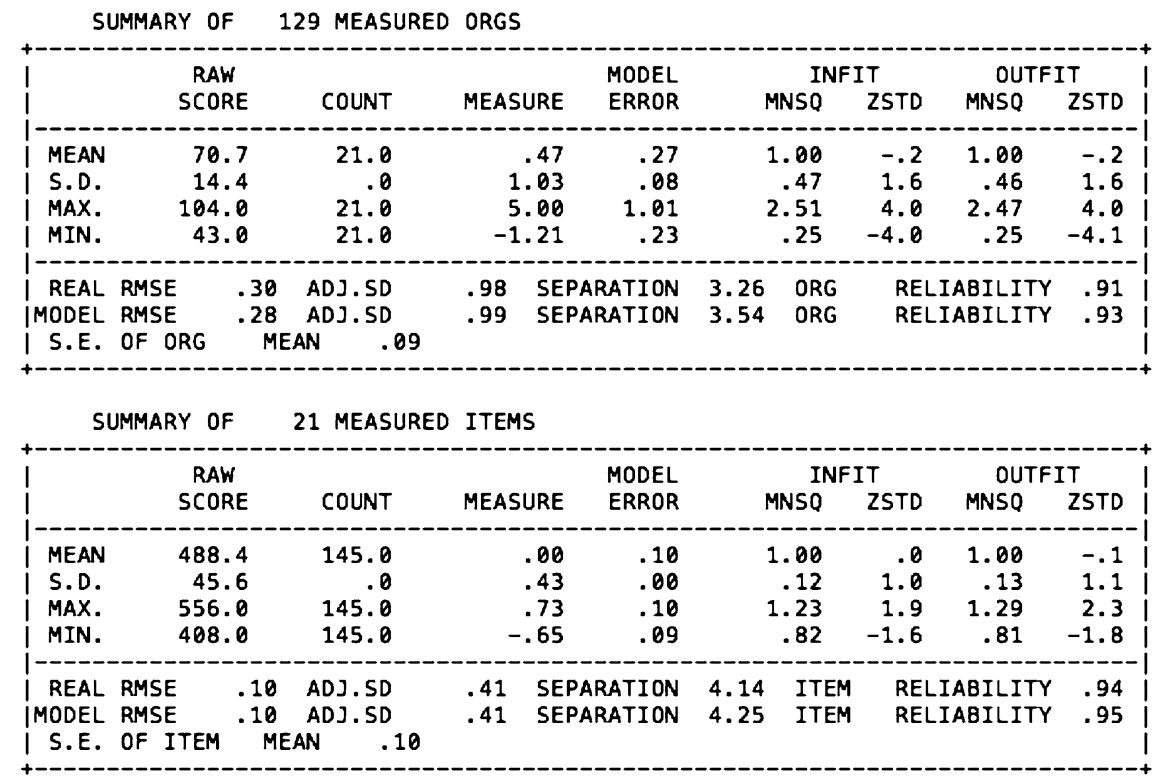

The subsequent Item-Response-Map shows the distribution of the participating hospitals and the relative difficulty of items. This illustration served as basis for determining the CMM for hospital process management as illustrated in Fig. 2. 


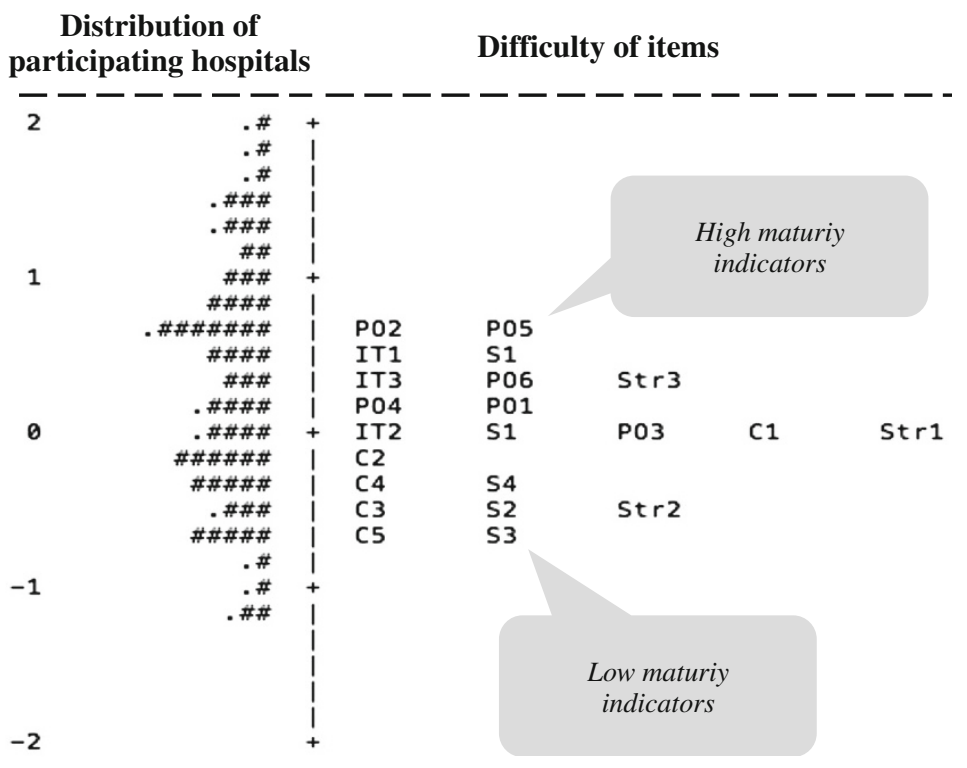

\section{References}

Ahern, Dennis M., Aaron Clouse, and Richard Turner. 2003. CMMI distilled: a practical introduction to integrated process improvement. Boston: Addison-Wesley.

Alibabaei, Ahmad, Bandara, Wasana, Aghdasi, Mohammad. 2009. Means of Achieving Business Process Management Success Factors. In 4th Mediterranean Conference on Information Systems (MCIS 2009), eds. Poulymenakou Angeliki, Pouloudi Nancy, Pramatari Katerinac, Athens: Athens University of Economics and Business.

Baskerville, Richard. 2008. What design science is not. European Journal of Information Systems 17(5): 441-443.

Becker, Jörg, Ralf Knackstedt, and Jens Pöppelbuß. 2009. Developing maturity models for IT management-a procedure model and its application. Business \& Information Systems Engineering 1(3): 213-222.

Bhattacherjee, Anol, and Neset Hikmet. 2007. Physicians' resistance toward healthcare information technology: a theoretical model and empirical test. European Journal of Information Systems 16: $725-737$.

Bond, Trevor G., and Christine M. Fox. 2007. Applying the Rasch Model, fundamental measurement in the human sciences. Mahwah: Lawrence Erlbaum Associates Publishers.

Bonham, Stephan S. 2008. Actionable strategies through integrated performance, process, project, and risk management. Boston: Artech House.

Bostrom, Robert P., and Stephen Heinen. 1977. MIS problems and failures-A socio-technical perspective. part ii-the application of the socio-technical theory. MIS Quarterly 1(4): 11-28.

Bragato, Laura, and Kerry Jacobs. 2003. Care pathways: the road to better health services? Journal of Health Organization and Management 17(3): 164-180.

Carter, Simon, and Lesley Henderson. 2005. Approaches to qualitative data collection in social science. In Handbook of health research methods, ed. Ann Bowling, and Shah Ebrahim, 215-229. Maidenhead: Open University Press.

Chiarini Tremblay, Monica, Alan R. Hevner, and J. Berndt Donald. 2010. Focus groups for artifact refinement and evaluation in design research. Communications of the Association for Information Systems 26: 599-618. 
Cleven, Anne, Robert Winter, and Peter Rohner. 2011. The performance impact of process orientation in hospitals: theoretical model and empirical test, Research Report, Institute of Information Management, University of St. Gallen.

Curtis, Bill, William E. Hefley, and Sally A. Miller. 2010. The people capability maturity modelguidelines for improving the workforce. Boston: Addison-Wesley.

Davenport, Thomas H. 1993. Process innovation-reengineering work through information technology. Boston: Harvard Business School Press.

Dekleva, Sasa, and David Drehmer. 1997. Measuring software engineering evolution: a Rasch calibration. Information Systems Research 8(1): 95-104.

de Ven, Van, H. Andrew, and Marshall Scott Poole. 1995. Explaining development and change in organizations. Academy of Management Review 20(3): 510-540.

Donaldson, Lex. 2006. The contingency theory of organizational design: challenges and opportunities. In Richard M. Burton, Bo Eriksen, Dorthe Døjbak Håkonsson and Charles C. Snow, eds., 19-40 New York: Springer US.

Dormont, Brigitte, Carine Milcent, Béatrice Desquins, and Jacques Huguenin. 2006. The Country of diversity: adjusting hospital payments for heterogeneity in Switzerland. Bulletin de la Société d'études Économiques et Sociales 64: 31-52.

Every, Nathan R., Judith Hochman, Richarda Becker, Steve Kopecky, and Christopher P. Cannon. 2000. Critical pathways: a review. Circulation 101: 461-465.

Fettke, Peter. 2006. State-of-the-Art des State-of-the-Art. Eine Untersuchung der Forschungsmethode "Review" innerhalb der Wirtschaftsinformatik. Wirtschaftsinformatik 48(4): 257-266.

Fisher, David M. 2004. The business process maturity model. A practical approach for identifying opportunities for optimization, BPTrends. http://www.bptrends.com/bpt/wp-content/publicationfiles/ 10-04\%20ART\%20BP\%20Maturity\%20Model\%20-\%20Fisher.pdf. Accessed 02.07.2014.

Fitterer, René, and Peter Rohner. 2010. Towards assessing the networkability of health care providers: a maturity model approach. Information Systems and e-Business Management 8(3): 309-333.

Frank, Ulrich. 2007. Evaluation of reference models, In Peter Fettke and Peter Loos, eds., 118-139. Idea Group Publishing: Hershey.

Fraser, Peter, Moultrie, James, and Gregory, Mike. 2002. The Use of Maturity Models/Grids as a Tool in Assessing Product Development Capability. In Proceedings of the IEEE International Engineering Management Conference (IEMC 2002). IEEE Engineering Management Society, 1: 244-249. Piscataway, NJ.

Gardner, John W. 1965. How to prevent organizational dry rot, Harper's Magazine 231: 20-26.

Gemmel, Paul, Darline Vandaelea, and Wim Tambeurc. 2008. Hospital process orientation (HPO): the development of a measurement tool. Total Quality Management \& Business Excellence 19(12): 1207-1217.

Goldstein, Susan Meyer, and Sharon B. Schweikhart. 2002. Empirical support for the Baldrige Award Framework in U.S. Hospitals. Health Care Management Review 27(1): 62-75.

Gregor, Shirley, and David Jones. 2007. The anatomy of a design theory. Journal of the Association for Information Systems 8(5): 312-335.

Hammer, Michael. 1990. Reengineering work—don't automate, Obliterate. Harvard Business Review 68(4): 104-112.

Hammer, Michael. 2010. Handbook on Business Process Management 1. In What is business process management?, ed. Jan vom Brocke, and Michael Rosemann, 3-16. Berlin: Springer.

Hammer, Michael, and James Champy. 1993. Reengineering the corporation-A manifesto for business revolution. New York: HarperCollins Publishers.

Hatch, Mary Jo. 1993. The dynamics of organizational culture. Academy of Management Journal 18(4): 657-693.

Helfert, Markus. 2009. Challenges of business process management in healthcare-experience in the Irish Healthcare Sector. Business Process Management Journal 15(6): 937-952.

Hellström, Andreas, Svante Lifvergren, and Johan Quist. 2010. Process management in healthcare: investigating why it's easier said than done. Journal of Manufacturing Technology Management 21(4): 499-511.

Herzlinger, Regina E. 2007. Who killed health care? America's \$2 trillion medical problem-and the consumer-driven cure. New York: Mc-Graw Hill.

Hevner, Alan R., Salvatore T. March, Jinsoo Park, and Sudha Ram. 2004. Design science in information systems research. MIS Quarterly 28(1): 75-105. 
Houy, Constantin, Peter Fettke, and Peter Loos. 2010. Empirical research in business process management-analysis of an emerging field of research. Business Process Management Journal 16(4): 619-661.

Hung, Richard Yu-Yuan. 2006. Business process management as competitive advantage: a review and empirical study. Total Quality Management \& Business Excellence 17(1): 21-40.

Hurst, Keith. 1996. The managerial and clinical implications of patient-focused care. Journal of Health Organization and Management 10(3): 59-77.

Killen, Catherine P., Robert A. Hunt, and Elko J. Kleinschmidt. 2008. Learning investments and organizational capabilities: case studies on the development of project portfolio management capabilities. International Journal of Managing Projects in Business 1(3): 334-351.

Klievink, Bram, and Marijn Janssen. 2009. Realizing Joined-up government-dynamic capabilities and stage models for transformation. Government Information Quarterly 26(2): 275-284.

Ko, Ryan K.L., Stephen S.G. Lee, and Eng Wah Lee. 2009. Business process management (BPM) standards: a survey. Business Process Management Journal 15(5): 744-791.

Kohlbacher, Markus, and Hajo A. Reijers. 2013. The effects of process-oriented organizational design on firm performance. Business Process Management Journal 19(2): 245-262.

Lahrmann, Gerrit, Marx, Frederik, Winter, Robert, and Wortmann, Felix. 2011. Business Intelligence Maturity: Development and Evaluation of a Theoretical Model. In Proceedings of the 44th Hawaii International Conference on System Sciences (HICSS-44), ed. R. Sprague. Los Alamitos: IEEE Computer Society.

Linacre, John M. and Benjamin D. Wright. 1998. Bigsteps Rasch Software, Version 2.82, http://www. winsteps.com/bigsteps.htm. Accessed 06.10.2010.

Lok, Peter, Richard Y. Hung, Paul Walsh, Paul Wang, and John Crawford. 2005. An integrative framework for measuring the extent to which organizational variables influence the success of process improvement programmes. Journal of Management Studies 42(7): 1357-1381.

March, Salvatore T., and Gerald F. Smith. 1995. Design and natural science research on information technology. Decision Support Systems 15(4): 251-266.

Marley, Kathryn A., David A. Collier, and Susan Meyer Goldstein. 2004. The role of clinical and process quality in achieving patient satisfaction in hospitals. Decision Sciences 35(3): 349-369.

Marshall, Martin N. 1996. Sampling for qualitative research. Family Practice 13(6): 522-525.

Marx, Frederik, Felix Wortmann, and Jörg H. Mayer. 2012. Ein Reifegradmodell für Unternehmenssteuerungssysteme. Wirtschaftinformatik 54(4): 189-204.

McCormack, Kevin, Jurgen Willems, Joachim van den Bergh, Dirk Deschoolmeester, Peter Willaert, Mojca Indihar Štemberger, Rok Škrinjar, Peter Trkman, Marcelo Bronzo Ladeira, Marcos Paulo Valadares, Vesna de Oliveira, Bosilj Vuksic, and Nikola Vlahovic. 2009. A global investigation of key turning points in business process maturity. Business Process Management Journal 15(5): 792-815.

McNulty, Terry, and Ewan Ferlie. 2004. Process transformation: limitations to radical organizational change within Public Service Organizations. Organization Studies 25(8): 1369-1412.

Mettler, Tobias. 2011a. Thinking in terms of design decisions when developing maturity models. International Journal of Strategic Decision Sciences 1(4): 76-87.

Mettler, Tobias. 2011b. Transformation of the hospital supply chain: how to measure the maturity of supplier relationship management systems in hospitals? International Journal of Healthcare Information Systems and Informatics 6(2): 1-13.

Mettler, Tobias and Rohner, Peter. 2009. An analysis of the factors influencing networkability in the healthcare sector. Health Services Management Research 22(4): 163-169.

Paulk, Mark C., Bill Curtis, Mary Beth Chrissis, and Charles V. Weber. 1993. Capability maturity model, Version 1.1. IEEE Software 10(4): 18-27.

Plattfaut, Ralf, Niehaves, Björn, Pöppelbuß, Jens, Becker, Jörg. 2011. Development of BPM Capabilities-Is Maturity the Right Path? In Proceedings of the 19th European Conference on Information Systems (ECIS 2011), eds. Tuunainen Virpi Kristiina, Nandhakumar Joe, Rossi Matti, Soliman W., paper 27. http://aisel.aisnet.org/ecis2011/27

Popovic, Ales, Pedro Simões Coelho, and Jurij Jaklič. 2009. The impact of business intelligence system maturity on information quality, Information Research, 14 (4), paper 417. http://InformationR.net/ir/ 14-4/paper417.html.

Raber, David, Wortmann, Felix, Winter, Robert:. 2013. Situational Business Intelligence Maturity Models: An Exploratory Analysis. In Proceedings of the 46th Hawaii International Conference on System Sciences 2013 (HICSS-46), 3797-3806. Los Alamitos: IEEE Computer Society. 
Reijers, Hajo A., and Selma Liman Mansar. 2005. Best practices in business process redesign: an overview and qualitative evaluation of successful redesign heuristics. Omega 33(4): 283-306.

Rohloff, Michael. 2009. Case Study and Maturity Model for Business Process Management Implementation. In 7th Business Process Management Conference (BPM 2009). LNCS, eds. Dayal Umeshwar, Eder Johann, Koehler Jana, Reijers Hajo A, 5701: 126-142. Berlin: Springer.

Rohner, Peter. 2012. Achieving impact with clinical process management in hospitals: an inspiring case. Business Process Management Journal 18(4/2012): 600-624.

Rosemann, Michael and Tonia de Bruin. 2005. Application of a holistic model for determining BPM maturity, BPTrends. http://bptrends.com-www.bptrends.com/publicationfiles/02-05\%20WP\% 20Application\%20of\%20a\%20Holistic\%20Model-\%20Rosemann-Bruin\%20-\%E2\%80\%A6.pdf. Accessed 02.07.2014.

Rosemann, Michael, and Jan vom Brocke. 2010. International Handbooks on Information Systems, 2010, Handbook on Business Process Management 1, Part I-Introduction, Methods, and Information Systems. In The six core elements of business process management, ed. Michael Rosemann, and Jan vom Brocke, 107-122. Berlin: Springer.

Scott, Tim, Russell Mannion, Huw Davies, and Martin N. Marshall. 2003. The quantitative measurement of organizational culture in health care: a review of the available instruments. Health Services Research 38(3): 923-945.

Skrinjar, Rok, Vesna Bosilj-Vuksic, and Mojca Indihar-Stemberger. 2008. The impact of business process orientation on financial and non-financial performance. Business Process Management Journal 14(5): 738-754.

Škrinjar, Rok, and Peter Trkman. 2013. Increasing process orientation with business process management: Critical practices'. International Journal of Information Management 33(1): 48-60.

Smart, P., Harry Maddern Andi, and Roger S. Maull. 2009. Understanding business process management: implications for theory and practice. British Journal of Management 20(4): 491-507.

Soanes, Catherine, and Angus Stevenson. 2008. Concise Oxford English Dictionary. New York: Oxford University Press Inc.

SwissDRG. 2011. SwissDRG system 1.0/2012. http://www.swissdrg.org/de/07_casemix_office/SwissDRG_ System_10.asp?navid=21. Accessed 5 Apr 2011.

Teece, David J., Gary Pisano, and Amy Shuen. 1997. Dynamic capabilities and strategic management. Strategic Management Journal 18(7): 509-533.

Trkman, Peter. 2010. The critical success factors of business process management. International Journal of Information Management 30(2): 125-134.

Van Looy, Amy. 2010. Does IT matter for business process maturity? A comparative study on business process maturity models. In On the move to meaningful internet systems: OTM 2010 workshops. LNCS, eds. Meersman Robert, Dillon Tharam, Herrero Pilar, 6428: 687-697. Berlin: Springer.

van Steenbergen, Marlies. 2011. Maturity and Effectiveness of Enterprise Architecture. Ph.D. thesis, Utrecht University, Utrecht.

Vera, Antonio, and Ludwig Kuntz. 2007. Process-based organization design and hospital efficiency. Health Care Management Review 32(1): 55-65.

vom Brocke, Jan, Simons, Alexander, Niehaves, Björn, Riemer, Kai, Plattfaut, Ralf, Cleven, Anne. 2009. Reconstructing the Giant: On the Importance of Rigour in Documenting the Literature Search Process. In Proceedings of the 17th European Conference on Information Systems (ECIS 2009), eds. Newell Sue, Whitley Edgar, Pouloudi Nancy, Wareham Jonathan, Mathiassen Lars, 2206-2217. http://is2.lse.ac.uk/asp/aspecis/20090183.pdf.

Vos, Leti, Robert J. van Oostenbrugge, Martien Limburg, Godefridus G. van Merode, and Siebren Groothuis. 2009. How to implement process-oriented care: a case study on the implementation of process-oriented in-hospital stroke care. Accreditation and Quality Assurance: Journal for Quality, Comparability and Reliability in Chemical Measurement 14(1): 5-13.

West, Elizabeth. 2001. Management matters: the link between hospital organisation and quality of patient care. Quality in Health Care 10: 40-48.

Zairi, Mohamed. 1997. Business process management-a boundaryless approach to modern competitiveness. Business Process Management Journal 3(1): 64-80. 